\title{
Diagnostic Utility of TLEI (Transducer-Like Enhancer of Split I) in Distinguishing Synovial Sarcoma from Mimicking Tumors
}

\author{
Madiha Bilal Qureshi' \\ Muhammad Usman Tariq ' \\ Jamshid Abdul-Ghafar (iD ${ }^{2}$ \\ Qurratulain Chundriger' \\ Nasir Ud Din' \\ 'Department of Pathology and \\ Laboratory Medicine, Aga Khan \\ University Hospital, Karachi, Pakistan; \\ ${ }^{2}$ Department of Pathology and Clinical \\ Laboratory, French Medical Institute for \\ Mothers and Children (FMIC), Kabul, \\ Afghanistan
}

Background: Synovial sarcoma (SS) is a high-grade spindle cell tumor that accounts for 5\% to $10 \%$ of soft tissue sarcomas. The majority originate from the deep intramuscular soft tissues of extremities with common sites including knee, ankle and feet. Immunohistochemical (IHC) stain TLE1 (transducer-like enhancer of split 1) is a potent diagnostic marker for distinguishing SS from mimicking tumors.

Methodology: The study was performed on 177 tumor cases, including 89 SS and 88 nonsynovial sarcoma (N-SS) cases which were diagnosed at Department of Pathology and Laboratory Medicine, Aga Khan University Hospital, from July 2019 to June 2020. Hematoxylin and eosin (H\&E) and IHC stained glass slides of these cases were reviewed. TLE1 expression was assessed based on the Remmele scoring system.

Results: Eighty-nine cases of SS and 88 cases of N-SS were included in the study. SS cases included 42 (47.2\%) monophasic subtype, 6 (6.7\%) biphasic subtype and 41 (46.1\%) poorly differentiated subtype. Major tumor types in N-SS cases were $27(30.7 \%)$ Ewing sarcoma (ES), 13 (14.8\%) leiomyosarcoma, 10 (11.4\%) undifferentiated sarcoma (US), 8 (9.1\%) fibrosarcomatous dermatofibrosarcoma protuberans and $7(8 \%)$ malignant peripheral nerve sheath tumor cases. Mean patients' age for SS cases was 26.14 years and for N-SS cases was 32.64 years. All 89 SS cases showed positive TLE1 expression. Out of 88 N-SS cases, 71 $(80.7 \%)$ were TLE1 negative and 17 (19.3\%) showed positive expression.

Conclusion: This study shows that TLE1 is a very sensitive and relatively specific IHC marker for SS. TLE1 expression can be observed in other soft tissue sarcomas but diffuse strong TLE1 expression is highly specific for SS. The diagnosis should not solely rely on TLE1 expression and morphologic features but should include soft tissue specific lineage markers to avoid misdiagnosis.

Keywords: synovial sarcoma, monophasic synovial sarcoma, TLE1

\section{Background}

Immunohistochemical (IHC) stain in soft tissue tumors is employed to detect the presence of lineage specific antigens in tumor cells. IHC stain is performed after thorough assessment of morphological features raising possibilities of differential diagnoses. Initial panel of antibodies determines broad lineage with additional panels specifying tumor type. Molecular studies provide an adjunctive tool in distinguishing specific tumors.

Synovial sarcoma (SS) is a high-grade spindle cell tumor that accounts for $5 \%$ to $10 \%$ of all soft tissue sarcomas. ${ }^{1}$ It can arise at any anatomic site in any age group, but
Correspondence: Jamshid Abdul-Ghafar Department of Pathology and Clinical Laboratory, French Medical Institute fo Mothers and Children (FMIC), Kabul, Afghanistan

Tel +93792827287

Email jamshid.jalal@fmic.org.af 
it most commonly involves the extremities in young adults. ${ }^{2}$ In the Pakistani population, the highest incidence has been observed in the 15-49 age group. ${ }^{3}$ Most of these tumors originate from the deep intramuscular soft tissues of extremities with common sites including knee, ankle, feet, and hand. Uncommon sites include the inguinal region, abdominal wall, and head and neck region. ${ }^{4}$

SS has three histologic subtypes; monophasic subtype comprises $50-60 \%$ cases. Tumor of this subtype is composed of monomorphic spindle-shaped cells arranged in sheets or fascicles. Mitotic figures are rarely appreciated. Differential diagnoses include malignant peripheral nerve sheath tumor (MPNST), fibrosarcomatous dermatofibrosarcoma protuberans (FS-DFSP), and some other spindle cell neoplasms. Biphasic subtype comprises of $20-30 \%$ cases of SS. Tumor of this subtype is composed of both spindle and epithelial components. Poorly differentiated subtype comprises $10-15 \%$ cases of SS and it is composed of diffuse sheets of small round blue cells with nuclear atypia, conspicuous nucleoli and high mitotic rate. The closest differential diagnosis of this subtype is Ewing sarcoma (ES). ${ }^{5}$

At the genetic level, SS is characterized by a specific translocation $\mathrm{t}(\mathrm{X} ; 18)(\mathrm{p} 11 ; \mathrm{q} 11)$. It is driven by fusion of SS18 gene on chromosome 18 with SSX gene on chromosome X (frequently SSX1 and SSX2, and rarely SSX4). ${ }^{6}$ Transducer-like enhancer of split 1 (TLE1) is a member of the TLE family of genes that encodes Groucho-like transcriptional corepressors and is one of the most frequently overexpressed genes in SS. It acts as transcriptional corepressor that inhibits the $\mathrm{Wnt} / \beta$-catenin signaling pathway. TLE1 binds other basic helix-loop-helix (bHLH) proteins causing repression of target genes. ${ }^{6}$ Hence, molecular studies through techniques like fluorescence in situ hybridization (FISH), reverse transcriptase-polymerase chain reaction (RT-PCR) and cytogenetics remain the gold standard for the diagnosis of SS. However, the use of these tests is limited due to lack of resources in developing and poor countries, such as Pakistan and Afghanistan.

IHC stains usually performed to establish the diagnosis of SS include TLE1, EMA, cytokeratins (CK AE1/AE3, CK7, CK19), CD99, CD34, BCL-2, and vimentin. Recent studies have demonstrated that TLE1 is the most sensitive and specific IHC marker for diagnosing and differentiating SS from histologic mimics. ${ }^{7}$

We conducted this study with an aim to assess the expression of TLE1 by IHC stain in SS and other neoplasms of variable lineages.

\section{Materials and Methods}

The study was conducted at the Section of Histopathology, Department of Pathology and Laboratory Medicine, Aga Khan University Hospital (AKUH). Incisional biopsies and surgical resection specimens of tumors on which the expression of TLE1 was assessed between July 2019 and June 2020 were included in the study. Twelve cases were positive for SYT fusion on FISH studies. Hematoxylin and eosin (H\&E) stained and IHC stained glass slides of these cases were retrieved and reviewed by two consultant histopathologists (MBQ, NUD). Exclusion criteria included autolyzed and poorly fixed tumors, trucut biopsies and the cases in which a definitive diagnosis could not be rendered on the basis of H\&E and IHC. TLE1 (Cell Marque TLE1 (1F5), Mouse Monoclonal Antibody, Cell Marque Corporation, Rocklin, USA) was used to stain tumor tissue. Positive and negative controls were run with every staining batch. Brown colored nuclear staining of tumor cell nuclei was assessed, scored and interpreted according to Remmele score (Remmele score $=$ Percentage of the stained tumor cells $\times$ Intensity of staining). Percentage was assessed as: 0 for no stain, 1 for $<10 \%, 2$ for $11-50 \%, 3$ for $51-80 \%$ and 4 for $81-100 \%$ of stained cells. Intensity of immunoreactivity was evaluated as: 0 for no stain, 1 for weak stain (faint light brown), 2 for moderate stain (dark brown staining of intensity less than that of positive control) and 3 for strong stain (dark brown nuclear staining equating intensity of positive control). Cumulative score was calculated as: $5-12=$ high, $3-4=$ moderate, $1-2=$ weak, and $0=$ negative. High and moderate score were interpreted as "positive" whereas weak score was interpreted as "negative".

Data regarding patients' demographics such as age and gender was available from patients' reports. Based on histological diagnoses, two categories were made; SS and non-synovial sarcoma (N-SS).

Data analysis was carried out via International Business Machines Statistical Package for the Social Sciences (IBM SPSS), version 21.0. Mean \pm SD was calculated for quantitative variables including patients' age and Remmele score. Frequency and percentages were determined for qualitative variables including gender, histological diagnosis (SS or N-SS) and TLE1 IHC expression. Sensitivity and specificity of TLE1 expression for SS were also calculated. Chi-square test was applied and $p$-value $\leq 0.05$ was interpreted as significant. 


\section{Results}

A total of 177 cases were included in the study. Patients' age ranged from 25-81 years. Eighty-nine (50.3\%) cases were diagnosed as SS and $88(49.7 \%)$ were diagnosed and categorized as N-SS. Among SS cases, 42 (47.2\%) were monophasic, $6(6.7 \%)$ were biphasic and 41 (46.1\%) were poorly differentiated subtype. The N-SS group included 27 (30.7\%) ES, 13 (14.8\%) leiomyosarcoma (LMS), 10 (11.4\%) undifferentiated sarcoma (US), 8 (9.1\%) FSDFSP and 7 (8\%) MPNST cases (Table 1).

Mean age of SS cases was 26.14 years with SD of \pm 7.47 years whereas mean age of N-SS cases was 32.64 years with SD of \pm 18.9 years. Forty-two (47.2\%) of the SS cases were male and 47 (52.8\%) were female. Fifty-eight (65.9\%) N-SS cases were male and $30(34.1 \%)$ were female.

All SS cases demonstrated positive TLE1 IHC expression. In contrast, TLE1 positivity was observed in $17(19.3 \%)$ $\mathrm{N}-\mathrm{SS}$ cases $(p$-value $=<0.001)$ (Table 2$)$. Among these N-SS cases, 4 (4.5\%) displayed strong TLE1 expression, 2 (2.2\%)

Table I Frequency of Different Tumors Stained with TLEI Antibody $(n=177)$

\begin{tabular}{|l|l|}
\hline Histological Diagnosis & Frequency (\%) \\
\hline Synovial sarcoma & \\
- Monophasic & $42(47.2 \%)$ \\
- Biphasic & $6(6.7 \%)$ \\
- Poorly differentiated & $4 \mathrm{I}(46.7 \%)$ \\
\hline Non-synovial sarcoma tumors & \\
- Ewing sarcoma & $20(30.7 \%)$ \\
- Leiomyosarcoma & $13(14.8 \%)$ \\
- Undifferentiated sarcoma & $10(11.4 \%)$ \\
- Fibrosarcomatous dermatofibrosarcoma & $8(9.1 \%)$ \\
- Mrotuberans & \\
- Embryonal rhabdomyosarcoma & $7(8 \%)$ \\
- Sarcomatoid carcinoma & $4(4.5 \%)$ \\
- Malignant solitary fibrous tumor & $4(4.5 \%)$ \\
- Epithelioid sarcoma & $3(3.4 \%)$ \\
- Malignant melanoma & $2(2.3 \%)$ \\
- Wilms tumor & $\mathrm{I}(\mathrm{I} .1 \%)$ \\
- Histiocytic sarcoma & $\mathrm{I}(\mathrm{I} .1 \%)$ \\
- Merkel cell carcinoma & $\mathrm{I}(\mathrm{I} .1 \%)$ \\
- Inflammatory myofibroblastic tumor & $\mathrm{I}(\mathrm{I} .1 \%)$ \\
\hline
\end{tabular}

Table 2 Comparison of Frequency of Overall TLEI IHC Expression in Synovial Sarcoma and Non-Synovial Sarcoma Cases $(n=177)$

\begin{tabular}{|l|c|c|c|}
\hline \multirow{2}{*}{ Histological Groups } & \multicolumn{2}{|c|}{ TLEI Expression } & \multirow{2}{*}{ p-value } \\
\cline { 2 - 3 } & Positive & Negative & \\
\hline Synovial sarcoma & $89(100 \%)$ & 0 & $<0.001$ \\
Non-synovial sarcoma & $17(19.3 \%)$ & $71(80.7 \%)$ & \\
\hline
\end{tabular}

displayed moderate expression and $11(12.5 \%)$ displayed weak expression $(p$-value $=<0.001)($ Table 3$)$.

The overall sensitivity and specificity calculated were $100 \%$ and $81 \%$, respectively. All subtypes including poorly differentiated subtype of SS demonstrated strong positive TLE1 expression (Figure 1A and B). ES, the closest differential of poorly differentiated SS, predominantly showed negative TLE1 expression (Figure 1C and D).

N-SS cases demonstrated variable strength and percentage of TLE1 IHC expression. The frequencies of pattern of expression (absent, weak, moderate and strong) have been summarized in Table 4. Strong positive expression was observed in single case each of ES, US, cellular schwannoma and malignant melanoma.

A single case of cellular schwannoma was stained with TLE1 antibody due to its increased cellularity, and it showed positive expression for TLE1 (Figure 2A and B). However, other markers of nerve sheath origin such as S100 and SOX10 were also positive in this case. Two cases of LMS demonstrated focal TLE1 staining of moderate intensity which was categorized as "weak" (Figure 2C and D).

A single case of malignant melanoma was stained with TLE1 antibody, and it showed strong diffuse positive TLE1 expression (Figure 3A and B). However, other IHC markers of melanocytic differentiation such as HMB45, Melan A, S100 and SOX10 were also positive in this case.

Weak positive expression was noticed in $3 \mathrm{ES}$ cases, 2 cases each of LMS and epithelioid sarcoma and 1 case each of US, MPNST, embryonal rhabdomyosarcoma and sarcomatoid carcinoma (Table 4).

\section{Discussion}

SS exhibits various morphologic patterns which show considerable overlap with other soft tissue sarcomas. $^{8}$ Monophasic SS shows fascicular arrangement of spindle shaped cells which brings MPNST, FS-DFSP, LMS, solitary fibrous tumor (SFT) and inflammatory myofibroblastic tumor in its differential diagnoses. Biphasic SS shows 
Table 3 Comparison of Frequency of Intensity of TLEI IHC Expression in Synovial Sarcoma and Non-Synovial Sarcoma Cases $(\mathrm{n}=$ 177)

\begin{tabular}{|l|c|c|c|c|c|}
\hline \multirow{2}{*}{ Histological Groups } & \multicolumn{4}{|c|}{ TLEI Expression } & \multirow{2}{*}{$p$-value } \\
\cline { 2 - 5 } & Negative & Weak Positive & Moderate Positive & Strong Positive & \\
\hline Synovial sarcoma & 0 & $2(2.2 \%)$ & $1(1.1 \%)$ & $86(96.7 \%)$ & $<0.00 \mathrm{I}$ \\
Non-synovial sarcoma & $7 \mathrm{I}(80.7 \%)$ & $\mathrm{II}(12.5 \%)$ & $2(2.2 \%)$ & $4(4.5 \%)$ & \\
\hline
\end{tabular}

glandular and spindle component which can also be seen in sarcomatoid carcinoma, Wilms tumor and others. Poorly differentiated is the most challenging type as it exhibits round cells with moderate to severe pleomorphism. Its differential diagnoses include ES and embryonal rhabdomyosarcoma which are also tumors of young children. ${ }^{9}$ Moreover, limited biopsy material including small incisional and trucut biopsies makes diagnosis more difficult.

In all such cases where there is morphologic overlap, accurate diagnosis requires immunomarkers. TLE1 has emerged as a highly specific and sensitive maker in distinguishing SS from its mimics. ${ }^{10}$ TLE1 genes encode human transcriptional corepressors that are engaged in embryogenesis and hematopoiesis. Gene expression studies have revealed overexpression of TLE1 in the nuclei of SS cells. ${ }^{11,12}$ This study evaluated TLE1 expression in SS and its histological mimics.

In contrast to $96 \%$ positive TLE1 expression observed by Knosel et al, ${ }^{13}$ this study showed $100 \%$ TLE1 expression in SS cases. In their study, 4 out of 14 cases of MPNST showed positive TLE1 expression, while this study showed only one out of 7 weak positive expression. All fibrosarcoma cases of both the studies were TLE1 negative. The specificity and sensitivity in that study was $96 \%$ and $75 \%$

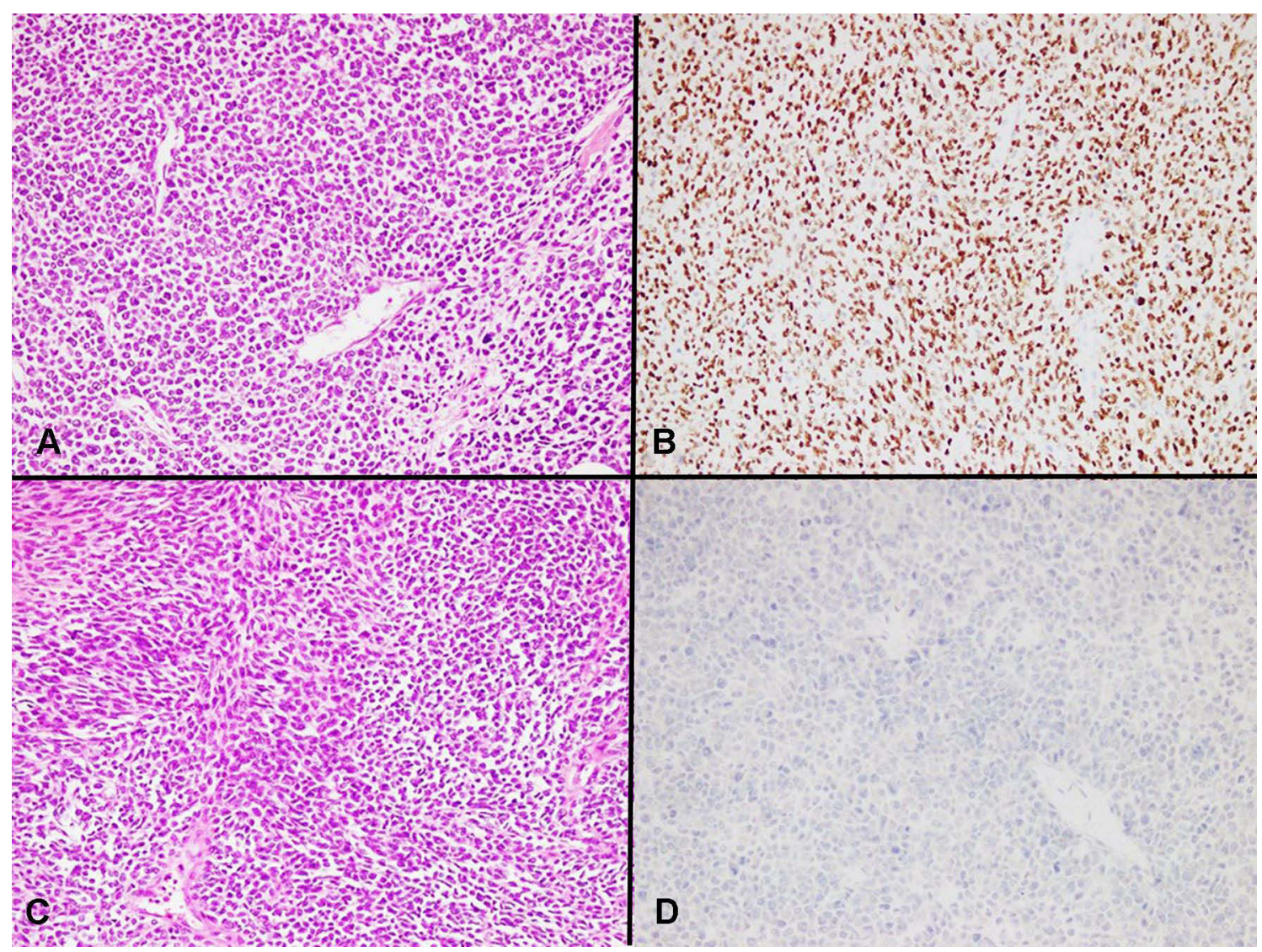

Figure I TLEI IHC staining in synovial sarcoma and Ewing sarcoma. (A) Poorly differentiated synovial sarcoma, H\&E, 20x. (B) Strong positive nuclear TLEI IHC expression in tumor cells, 20x. (C) Ewing sarcoma, H\&E, 20x. (D) Negative TLEI IHC expression in cells of Ewing sarcoma tumor cells, 20x. 
Table 4 Summary of Intensity-Wise TLEI Expression in Non-Synovial Sarcoma Entities Showing Positive TLEI Expression

\begin{tabular}{|c|c|c|c|c|c|}
\hline \multirow[t]{2}{*}{ Histological Diagnosis } & \multirow[t]{2}{*}{ No. of Cases Stained } & \multicolumn{4}{|c|}{ TLEI Expression } \\
\hline & & Negative & Weak Positive & Moderate Positive & Strong Positive \\
\hline Ewing sarcoma & 27 & 22 & 3 & I & I \\
\hline Leiomyosarcoma & 13 & 11 & 2 & - & - \\
\hline Embryonal rhabdomyosarcoma & 4 & 2 & I & 1 & - \\
\hline Epithelioid sarcoma & 2 & - & 2 & - & - \\
\hline Undifferentiated sarcoma & 10 & 8 & 1 & - & 1 \\
\hline Malignant melanoma & I & - & - & - & 1 \\
\hline Sarcomatoid carcinoma & 4 & 3 & I & - & - \\
\hline Malignant peripheral nerve sheath tumor & 7 & 6 & 1 & - & - \\
\hline Cellular schwannoma & I & - & - & - & 1 \\
\hline
\end{tabular}

respectively as compared to $81 \%$ and $100 \%$ in this study which may be attributed to more varied tumor collection in this study.

In a study conducted by Foo et al ${ }^{14}$ TLE1 positivity was observed in 4 out of 49 cases of SFT in contrast to negativity in all 3 SFT cases of this study. Interestingly, in comparison to all 23 ES negative cases in Foo et al's study and another study, ${ }^{14,15}$ this study displayed TLE1 positive expression in 5 out of 27 cases, 3 of them were weak and one case each demonstrated moderate and strong expression. FS-DFSP

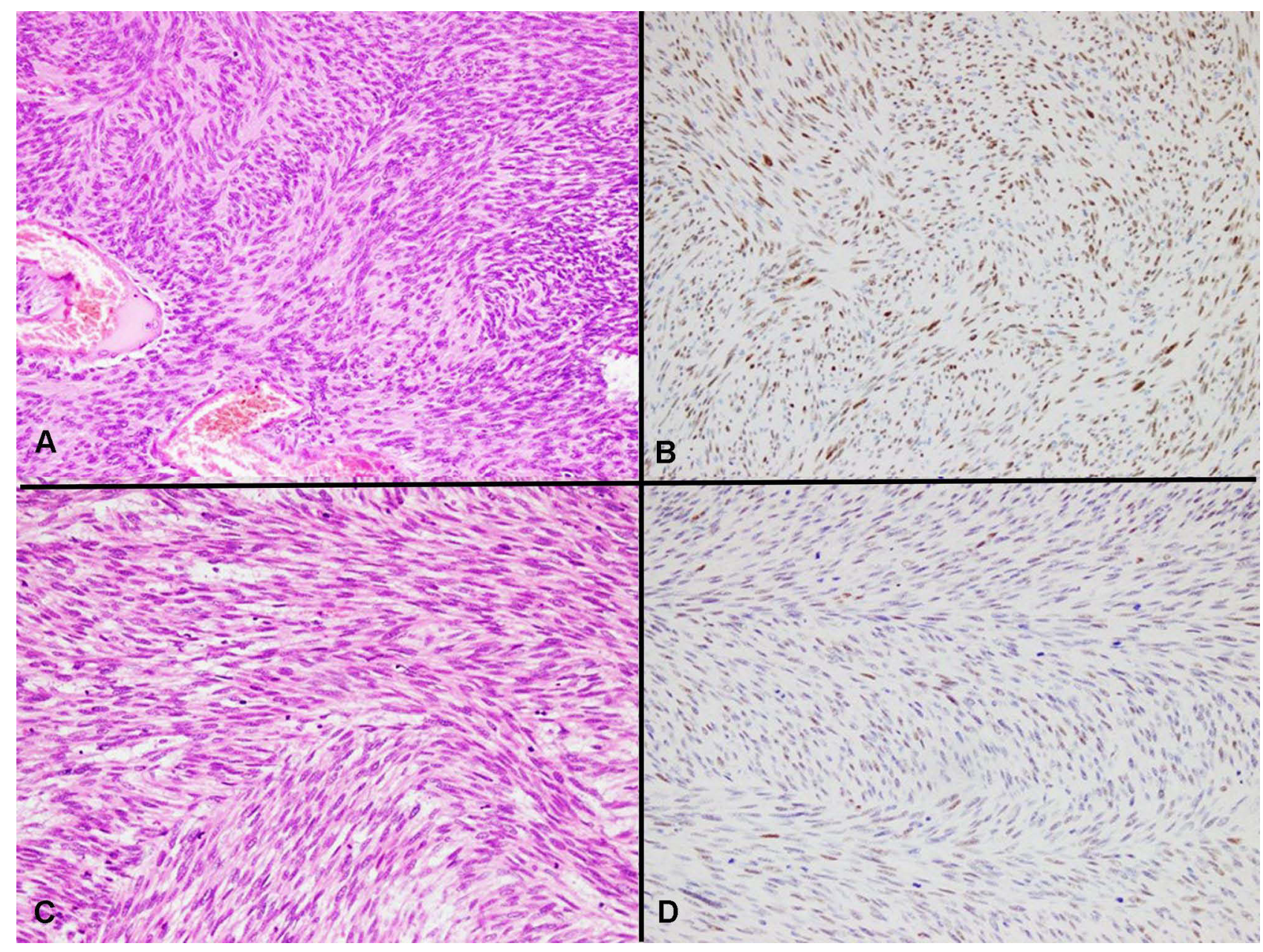

Figure 2 TLEI IHC staining in cellular schwannoma and leiomyosarcoma. (A) Cellular schwannoma, H\&E, 20x. (B) Strong nuclear TLEI expression in tumor cells of cellular schwannoma, 20x. (C) Leiomyosarcoma, H\&E, 20x. (D) Moderate nuclear TLEI expression in tumor cells of leiomyosarcoma, 20x. 


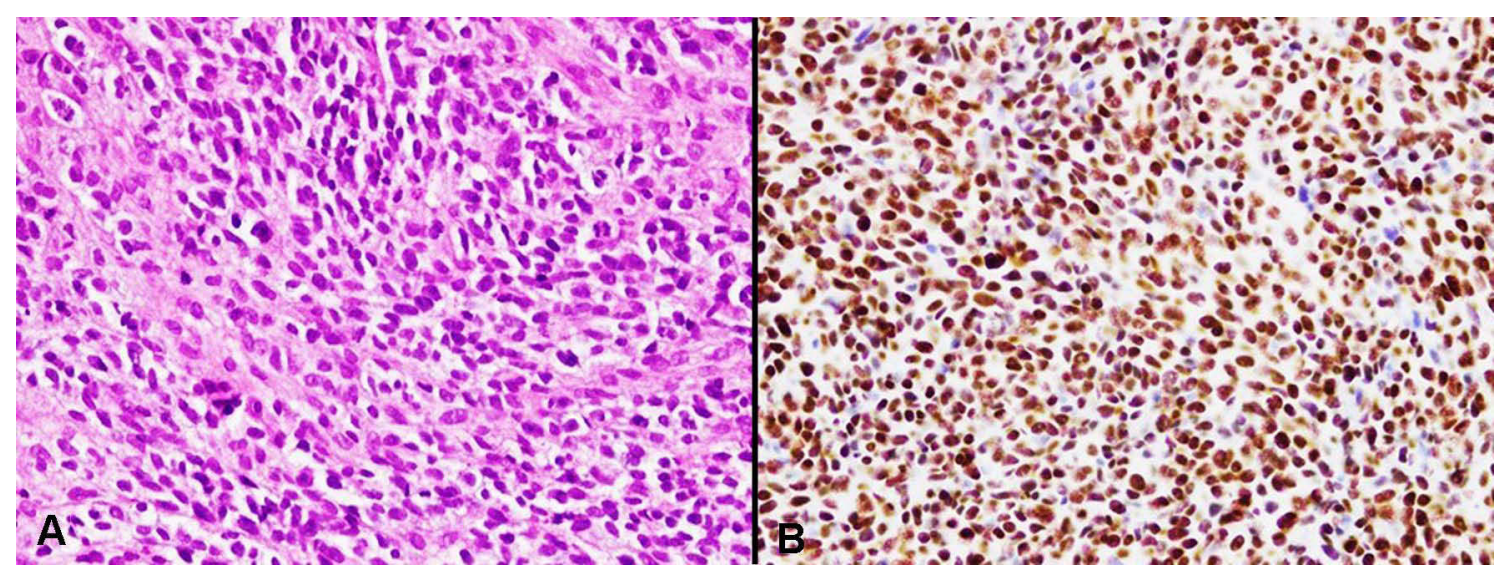

Figure 3 TLEI staining in malignant melanoma. (A) Malignant melanoma, H\&E, 20x. (B) Strong nuclear expression of TLEI IHC stain in melanoma cells, 20x.

cases of both studies were TLE1 negative. In a study by Chuang et al, 7 SS cases out of 50 were TLE1 negative. Furthermore, all the cases of rhabdomyosarcoma $(n=3)$ and LMS $(\mathrm{n}=5)$ were TLE1 negative. ${ }^{16}$ On the contrary, we observed 2 weak positive LMS cases out of total 13 and 2 positive cases of rhabdomyosarcoma out of 4 cases.

The limitations in the current study include retrospective nature and limited number of each N-SS entity stained with TLE1 antibody. Lack of financial resources also limits molecular confirmation of these cases. Nevertheless, histological features combined with IHC stains such as CKAE1/AE3, EMA and TLE1 have a great yield in the diagnosis of SS, however, it is important to have sound knowledge of other entities displaying TLE1 expression in order to avoid misdiagnosis.

\section{Conclusion}

This study shows that TLE1 is a highly sensitive and relatively specific IHC marker for establishing SS diagnosis. The expression in SS cases is usually diffuse and strong. However, its expression in other tumors can be misleading. Therefore, correlation with clinical, histological features and expression of other IHC markers is necessary for making the correct diagnosis.

\section{Abbreviations}

TLE1, transducer-like enhancer of split 1; IHC, immunohistochemistry; SS, synovial sarcoma; N-SS, non-synovial sarcoma; H\&E, hematoxylin and eosin; ES, Ewing sarcoma; US, undifferentiated sarcoma; FS-DFSP, fibrosarcomatous dermatofibrosarcoma protuberans; MPNST, malignant peripheral nerve sheath tumor; SFT, solitary fibrous tumor; LMS, leiomyosarcoma; FISH, fluorescence in situ hybridization; RT-PCR, reverse transcriptasepolymerase chain reaction; $\mathrm{CK}$, cytokeratin; AKUH, Aga Khan University Hospital.

\section{Data Sharing Statement}

Data and materials of this work are available from the corresponding author on reasonable request.

\section{Ethics Approval and Consent to Participate}

All procedures performed on patient tumor samples in this study were in accordance with the ethical standards of the Institute Ethics Committee (ERC) and with the 1964 Helsinki Declaration and its later amendments or comparable ethical standards. The study was approved by ERC of AKU (3516- Pat-ERC-15). Informed consent was obtained in patients in whom follow up was available.

\section{Consent for Publication}

Written informed consent was obtained.

\section{Author Contributions}

All authors made substantial contributions to conception and design, acquisition, analysis and interpretation of data; took part in drafting the article and revising it critically for important intellectual content; agreed to submit to the current journal; gave final approval of the version to be published; and agree to be accountable for all aspects of the work.

\section{Funding}

No financial support was provided for this study. 


\section{Disclosure}

It is declared that all authors have no conflicts of interest for this work.

\section{References}

1. Thway K, Fisher C. Synovial sarcoma: defining features and diagnostic evolution. Ann Diagn Pathol. 2014;18:369-380. doi:10.1016/j. anndiagpath.2014.09.002

2. Fisher C. Synovial sarcoma. Ann Diagn Pathol. 1998;2:401-421. doi:10.1016/S1092-9134(98)80042-7

3. Bhurgri Y, Bhurgri H, Pervez S, et al. Epidemiology of soft tissue sarcomas in Karachi South, Pakistan (1995-7). Asian Pac J Cancer Prev. 2008;9:709-714.

4. Cadman NL, Soule EH, Kelly PJ. Synovial sarcoma; an analysis of 134 tumors. Cancer. 1965;18(5):613-627. doi:10.1002/1097-0142(196505)18:5<613::aid-cncr2820180510>3.0.co;2-v

5. WHO Classification of Tumours Editorial Board. Soft tissue and bone tumours. Lyon (France): International Agency for Research on Cancer; 2020. Accessed November 11, 2021. Available from: https://publica tions.iarc.fr/588.

6. Su L, Sampaio AV, Jones KB, et al. Deconstruction of the SS18-SSX fusion oncoprotein complex: insights into disease etiology and therapeutics. Cancer Cell. 2012;21:333-347. doi:10.1016/j. ccr.2012.01.010

7. Terry J, Saito T, Subramanian S, et al. TLE1 as a diagnostic immunohistochemical marker for synovial sarcoma emerging from gene expression profiling studies. Am J Surg Pathol. 2007;31:240-246. doi:10.1097/01.pas.0000213330.71745.39

8. Fisher C. Immunohistochemistry in diagnosis of soft tissue tumors. Histopathology. 2011;58:1001-1012. doi:10.1111/j.1365-2559.2010. 03707.x
9. Jain S, Xu R, Prieto VG, Lee P. Molecular classification of soft tissue sarcomas and its clinical applications. Int $J$ Clin Exp Pathol. 2010;3:416-428

10. Qureshi MB, Uddin N, Tariq M, Raheem A, Pervez S. Frequency of transducer-like enhancer of split 1 immunohistochemical expression in synovial sarcoma: an institution-based cross-sectional study. Cureus. 2019;11:6357. doi:10.7759/cureus.6357

11. Vlenterie M, Hillebrandt-Roeffen MH, Flucke UE, et al. Next generation sequencing in synovial sarcoma reveals novel gene mutations. Oncotarget. 2015;6:34680-34690. doi:10.18632/oncotarget.5786

12. El Beaino M, Jupiter DC, Assi T, et al. Diagnostic value of TLE1 in synovial sarcoma: a systematic review and meta-analysis. Sarcoma. 2020;2020:1-6. doi:10.1155/2020/7192347

13. Knosel T, Heretsch S, Altendorf-Hofmann A, et al. TLE1 is a robust diagnostic biomarker for synovial sarcomas and correlates with $\mathrm{t}(\mathrm{X}$; 18): analysis of 319 cases. Eur J Cancer. 2010;46:1170-1176. doi:10.1016/j.ejca.2010.01.032

14. Foo WC, Cruise MW, Wick MR, Hornick JL. Immunohistochemical staining for TLE1 distinguishes synovial sarcoma from histologic mimics. Am J Clin Pathol. 2011;135:839-844. doi:10.1309/ AJCP45SSNAOPXYXU

15. Jagdis A, Rubin BP, Tubbs RR, Pacheco M, Nielsen TO. Prospective evaluation of TLE1 as a diagnostic immunohistochemical marker in synovial sarcoma. Am J Surg Pathol. 2009;33:1743-1751. doi:10.1097/PAS.0b013e3181b7ed36

16. Chuang HC, Hsu SC, Huang CG, Hsueh S, Ng KF, Chen TC. Reappraisal of TLE-1 immunohistochemical staining and molecular detection of SS18-SSX fusion transcripts for synovial sarcoma. Pathol Int. 2013;63:573-580. doi:10.1111/pin.12113
International Journal of General Medicine

\section{Publish your work in this journal}

The International Journal of General Medicine is an international, peer-reviewed open-access journal that focuses on general and internal medicine, pathogenesis, epidemiology, diagnosis, monitoring and treatment protocols. The journal is characterized by the rapid reporting of reviews, original research and clinical studies

\section{Dovepress}

across all disease areas. The manuscript management system is completely online and includes a very quick and fair peer-review system, which is all easy to use. Visit http://www.dovepress.com/ testimonials.php to read real quotes from published authors. 